\title{
Nanoscale surface characterization using laser interference microscopy
}

\author{
Pavel S. Ignatyev ${ }^{1, *}$, Andrey A. Skrynnik ${ }^{1}$, and Yury A. Melnik ${ }^{2}$ \\ 1 Joint stock company "Urals optical and mechanical plant", Vostochnaya str. 33b, Ekaterinburg 620100, Russia \\ 2 Moscow State University of Technology "STANKIN", Vadkovsky per. 1, Moscow 127994, Russia
}

Received: 21 September 2017 / Accepted: 11 December 2017

\begin{abstract}
Nanoscale surface characterization is one of the most significant parts of modern materials development and application. The modern microscopes are expensive and complicated tools, and its use for industrial tasks is limited due to laborious sample preparation, measurement procedures, and low operation speed. The laser modulation interference microscopy method (MIM) for real-time quantitative and qualitative analysis of glass, metals, ceramics, and various coatings has a spatial resolution of $0.1 \mathrm{~nm}$ for vertical and up to $100 \mathrm{~nm}$ for lateral. It is proposed as an alternative to traditional scanning electron microscopy (SEM) and atomic force microscopy (AFM) methods. It is demonstrated that in the cases of roughness metrology for super smooth $(R a>1 \mathrm{~nm})$ surfaces the application of a laser interference microscopy techniques is more optimal than conventional SEM and AFM. The comparison of semiconductor test structure for lateral dimensions measurements obtained with SEM and AFM and white light interferometer also demonstrates the advantages of MIM technique.
\end{abstract}

Keywords: Laser interference microscopy / quantitative phase imaging / super-resolution / nanometrology / microelectronics

\section{Introduction}

Since 2015, a new class of quantitative phase imaging methods was officially approved. This class brings together methods of white light interferometry $[1,2]$, phase shift interferometry [3-5], digital holographic microscopy [6,7], laser interference microscopy $[8,9]$, coherent phase microscopy [10], coherent correlation interferometry [11,12], etc.

The main advantage of methods above is an ability to reconstruct the quantitative topological phase relief $[13,14]$ which combines optical and geometrical properties of an investigated sample. It is also known that some quantitative phase imaging methods demonstrate lateral superresolution up to $100 \mathrm{~nm}$.

In this study was concluded that quantitative phase imaging methods are the worthy alternative to traditional methods of optical, probe and electron microscopy methods, or extra tool for additional samples characterization.

It is also discussed new results of a laser interference microscopy methods application for actual problems of optical industry, microelectronics, and biomedical investigations $[8,9]$.

\section{Materials and methods}

The MIM-340 laser interference microscope [9] with modified illuminator scheme was used as experimental setup. Calculation of the object phase shifts is made by modified phase steps method:

$$
\left\{\begin{array}{l}
I_{0}(x, y)=A(x, y)+B(x, y) \cos (\Phi(x, y)) \\
I_{1}(x, y)=A(x, y)+B(x, y) \cos (\Phi(x, y+k d)) \\
I_{2}(x, y)=A(x, y)+B(x, y) \cos (\Phi(x, y+2 k d)) \\
I_{3}(x, y, t)=A(x, y)+B(x, y) \cos (\Phi(x, y+3 k d(t)))
\end{array}\right.
$$

where $I_{n}(x, y)$ is the distribution of the intensity of the photosensor field of view, $k$ is wave number, $d$ is displacement of the reference mirror.

The sought value of the difference of phases $\Phi$ is to be determined using the following equation:

$$
\begin{gathered}
\Phi(x, y)=\text { arctg } \\
{\left[\frac{\sqrt{\left[\left(I_{1}-I_{2}\right)+\left(I_{0}-I_{3}\left(t_{0}\right)\right)\right] \cdot\left[3\left(I_{1}-I_{2}\right)-\left(I_{0}-I_{3}\left(t_{0}\right)\right)\right]}}{I_{1}+I_{2}-I_{0}-I_{3}\left(t_{0}\right)}\right]}
\end{gathered}
$$

\footnotetext{
* e-mail: shvabe.nano@gmail.com
} 


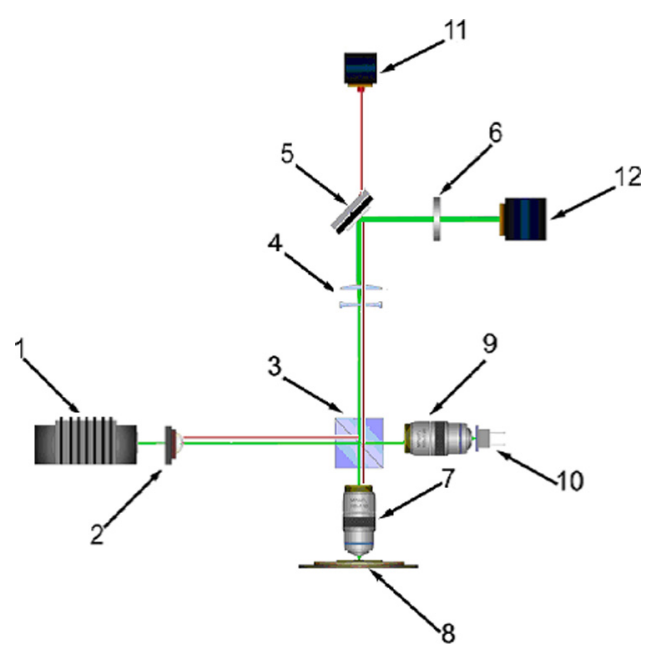

Fig. 1. Optical diagram of microscope.

where $I_{3}\left(t_{0}\right)$ is the value of the intensity determined by the photosensor exposition time.

With such variant, the error of calculation of the of the phase difference in each point of the image $I_{0}(x, y)-I_{3}(x, y)$ is reduced with minimal intensity due to the registration of the interferential signal variable component by analog with the above described time intervals method. The standing points (value of the support mirror shift) $d$ and of the law of displacement $d(t)$ of the reference mirror are also chosen to proceed from the minimization of the phases difference determination error.

The Microscope optical system represents a device in which there are realized two optical channels: navigational channel (white light channel) and measuring channel. Both channels give a possibility to use the instrument in two different modes: navigational (search of a microstructure of the material testing sample for study) and the measuring one.

The optical diagram of the Microscope optical system is given in Figure 1, the optical path of the navigational channel is shown in red lines, the optical path of measuring channel is shown in green lines.

The navigational channel represents the far-field optical microscope on reflected light is used to search the region of interest and to document the survey frame. The radiation source is a LED 2 that illuminates the object through the beam splitter 3 . The object image via the tube lens 4 is projected to the digital camera 11 then it comes to the PC. The measuring channel is intended to study the micro-object relief and to get the image with nanoscale resolution; it represents the micro-interferometer according to the Linnik scheme with phase modulator 10 in the reference arm. The semiconductor laser 1 with wavelength $405 \mathrm{~nm}$ is used as a coherent light source. The collimated laser beam 1 is divided by the beam splitter 3 into two beams. One of the beams (objective) is focused by the micro objective 13 of the objective arm 7 on the test object 8 and after reflection from the object. It passes the light splitter 3 and projecting system, consisting of the tube lens 4 and projective 6 , comes to the photosensor of the digital camera 12. The reference beam is focused by the micro objective of

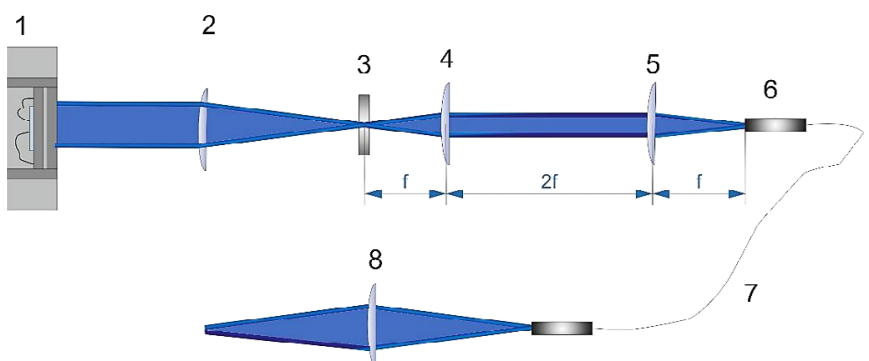

Fig. 2. MIM-340 modified illuminator optical diagram.

support arm 9 on the mirror of phase modulator 10 that effects the linear-periodical modulation of the optical difference, and after reflection from the mirror of the phase modulator 10 also comes to the photosensor of the digital camera 12 , then comes to the PC. The specially developed software realizes automatized processing of interferograms and displays of investigation results on the $\mathrm{PC}$ monitors in the form of three-dimensional and two-dimensional profiles of the object, graphics, and histograms. The selection of the mode of the Microscope optical system operation (navigational or measuring) is made with the help of the deflecting mirror 5 controlled by the user from the PC.

The MIM-340 modified illuminator optical diagram is presented in Figure 2. The modified illuminator is used to reduce coherent noises in MIM imaging optics. It is known that coherent noises cause an additional parasitic relief of $10-15 \mathrm{~nm}$; it is not sufficient for profile measurement of $100 \mathrm{~nm}$ and higher. But this relief is unacceptable for super smooth surfaces roughness measurements [12]. The modified illuminator allows to minimize coherent noises level up to $<1 \mathrm{~nm}$ level.

\section{Discussion of results}

Super smooth surfaces roughness measurement is one of the actual problems of optical industry and microelectronics $[15,16]$. The roughness of laser mirror surface determines directly the laser beam scattering which leads to energy losses in a resonator. Modern laser mirrors for UV and visible lasers have roughness $R a>1 \mathrm{~nm}$, and to measure such small roughness values, the specific microscopy methods are necessary [17].

The traditional atomic force microscopy (AFM) is the widely applicable tool or such measurements, but there are some difficulties in metrology, for example, in low repeatability of laser mirrors dielectric coating Ra measurements. The problem is in atomic level interactions of charged cantilever tip with relatively soft nanoscale surface even in non-contact modes [18]. This interaction causes the trace-retrace difference of single scan and low repeatability of measurements.

The second tool which engineers used for this task is white light interferometry [2]. The traditional optical profilometry is free of cantilever interaction problem, but the metrology is also unstable due to low lateral resolution. It means that all relief inside single diffraction limited point spread function PSF $(350 \mathrm{~nm}$ for the $100 \times / 0.85$ objective lens) is averaged. One more averaging was done 
Table 1. The laser mirror roughness measurements.

\begin{tabular}{lccl}
\hline Parameter & AFM & WLI & MIM-340 \\
\hline $\begin{array}{l}\text { Laser mirror substrate } \\
\text { Roughness RMS, nm }\end{array}$ & 1.6 & 0.9 & 1.8 \\
Uncertainty, nm & 0.2 & 0.5 & 0.4 \\
Laser mirror & & & \\
Roughness RMS, nm & 1.5 & 0.9 & 1.7 \\
Uncertainty, nm & 0.2 & 0.4 & 0.3 \\
\hline
\end{tabular}

for up to $5 \mathrm{~nm}$ spectral line width and microscope selfnoises compensation. Finally, the results of such measurements are quite acceptable, but the uncertainty level for $R a=1 \mathrm{~nm}$ is $0.3-0.5 \mathrm{~nm}$ make this measurement too rough for scattering losses estimations.

In this case application of super-resolution [19], quantitative phase imaging methods are more promising due to high lateral resolution, operating speed and absent of any mechanical electrical or magnetic interactions with measured surface $[11,14]$.

The comparison of laser mirror substrate and dielectric laser mirror roughness measurements using AFM (Veeco Dimensions 5), white light interferometer (WLI) (Zygo Newview 5000), and modified MIM-340 is demonstrated in Table 1. For all measurements the same mirror and substrate were used, scan size for AFM is $20 \times 20 \mu \mathrm{m}$, field of view for WLI was $250 \times 250 \mu \mathrm{m}$ for the $50 \times / 0.6$ lens with ROI of $20 \times 20 \mu \mathrm{m}$, field of view for MIM was $20 \times 18 \mu \mathrm{m}$ for the $50 \times / 0.8$ lens.

The difference in roughness could be explained by the fact that multilayer refractive coating makes the surface smoother, especially in nanoscale roughness range [20]. The uncertainty values difference for WLI and MIM caused by reflection ratio difference of substrate $(<1 \%$ reflection) and mirror ( $10 \%$ for $\lambda=532 \mathrm{~nm})$. The substrate of crystalline glass-ceramic material (sitall) and mirror for $633 \mathrm{~nm}$ spectral range have been used for this experiment [21].

Linear dimensions measurements for microelectronics samples is also the actual task in the semiconductor industry [22-25]. The essence of this problem consists in requirements of lateral dimensions measurements uncertainty of $3 \sigma<50 \mathrm{~nm}$, according to so-called semi-standards for analog microelectronics. It could be easily achieved in laboratories with AFM and SEM but industrial application AFM and SEM as the in-line tool is limited due to high cost and of low operating speed measurements. That is why optical measurement tools seem more promising for the inline metrology; the traditional WLI methods meet the requirements of low cost and high operating speed it also widely uses for vertical dimensions measurements and provides $<1 \mathrm{~nm}$ uncertainty $[26,27]$.

Table 2 demonstrates the results of lateral dimensions measurements experiment. The test sample which consists of different (from 1.0 to $3.0 \mu \mathrm{m}$ ) width stripes was prepared to estimate the uncertainty of lateral dimensions measurements for AFM, SEM, WLI, and MIM.

The SEM (JSM-IT100 InTouchScop), AFM (Veeco Dimensions 5), WLI (Zygo Newview 5000), and modified MIM-340 were used for experiments [9]. For all measure-
Table 2. Results of lateral dimensions measurements experiment.

\begin{tabular}{lllll}
\hline Parameter & SEM & AFM & WLI & MIM-340 \\
\hline 1.0 mm Stripe & & & & \\
Measured value, $\mu \mathrm{m}$ & 1.0 & 1.0 & 1.2 & 1.1 \\
$\begin{array}{l}\text { Uncertainty, } \mu \mathrm{m} \\
1.2 \mu \mathrm{m} \text { Stripe }\end{array}$ & 0.05 & 0.10 & 0.5 & 0.10 \\
Measured value, $\mu \mathrm{m}$ & 1.2 & 1.2 & 1,3 & 1.2 \\
Uncertainty, $\mu \mathrm{m}$ & 0.05 & 0.10 & 0.50 & 0.10 \\
$2.0 \mu \mathrm{m}$ Stripe & & & & \\
Measured value, $\mu \mathrm{m}$ & 2.0 & 2.0 & 2.2 & 2.0 \\
Uncertainty, $\mu \mathrm{m}$ & 0.05 & 0.10 & 0.50 & 0.15 \\
$\begin{array}{l}\text { 3.0 } \mu \mathrm{m} \text { Stripe } \\
\text { Measured value, } \mu \mathrm{m}\end{array}$ & 3.0 & 3.0 & 3.1 & 3.0 \\
Uncertainty, $\mu \mathrm{m}$ & 0.05 & 0.10 & 0.50 & 0.1 \\
\hline
\end{tabular}

ments same sample and structure were used, scan size for AFM was $20 \times 20 \mu \mathrm{m}$, field of view for WLI is $250 \times 250 \mu \mathrm{m}$ for the $50 \times / 0.6$ lens, field of view for MIM was $20 \times 18 \mu \mathrm{m}$ for the $50 \times / 0.8$ lens.

The uncertainty for this experiment was estimated as composition of random standard uncertainty and systematic standard uncertainty.

The random standard uncertainty is characterized by RMS of stripe width $x_{i}$, using the following equation:

$$
S(x)=\sqrt{\frac{\sum\left(x_{i}-x\right)^{2}}{(n-1)}},
$$

where $x$ is the RMS of measurement results, $\mathrm{n}$ is its number.

The systematic standard uncertainty $\theta$ is characterized by measurement device and estimated with the following equation:

$$
\theta=K \sqrt{\sum_{i=1}^{n} \theta_{i}^{2}},
$$

where $\theta$ is the confidence limit of systematic standard uncertainty, $\theta_{i}$ are the limits of $i$ systematic standard uncertainty, $K$ is the coefficient, $K=1.1$ for $P=0.95$.

The confidence limits of systematic standard uncertainty for measurement devices estimated with the following equation:

$$
\varepsilon=t S(\tilde{x})
$$

where $t$ is Student's coefficient [28] $t=2,1$ for $n=20$ measurements. $S(\tilde{x})$ is the uncertainty of test- structure estimated with SEM.

The composition of random standard uncertainty and systematic standard uncertainty is calculated with the following equation:

$$
\Delta=K S_{\Sigma}
$$


where $K$ is the coefficient dependent on the relation of random standard uncertainty and systematic standard uncertainty:

$$
K=\frac{\varepsilon+\theta}{S(x)}+\sqrt{\sum_{i=1}^{m} \frac{\theta_{i}^{2}}{3}} .
$$

The RMS of the measurements results is calculated as:

$$
S_{\Sigma}=\sqrt{\sum_{i=1}^{m} \frac{\theta_{i}^{2}}{3}+S^{2}(x)} .
$$

The comparison of stripes width measured values and uncertainties reveal the advantages of SEM and AFM methods as multipurpose tools. WLI lateral dimensions measurements demonstrate nonacceptable uncertainty of $40-50 \%$ due to the low numeric aperture (NA) of interferometric objectives [29].

\section{Conclusion}

The super smooth surfaces roughness measurements and micro- and nanostructure linear dimensions measurements are the two main tasks in optical and microelectronic industries. Is shown that more than $90 \%$ of such experiments, could be carried out in laboratories using traditional AFM and SEM methods, but industrial tasks make these methods nonacceptable due to high cost and low operating speed. That is why optical measurement tools seem more promising for the in-line metrology. The traditional WLI methods meet the requirements of relatively cheap and high operating speed; it also widely uses for vertical dimensions as well as roughness measurements and provides $<1 \mathrm{~nm}$ uncertainty, but for lateral dimensions measurements it demonstrate nonacceptable uncertainty of $40-50 \%$ due to the low NA of interferometric objectives.

The MIM microscopes are proposed as alternative to WLI methods. MIM technology demonstrates the combination of optical, software and design solutions to achieve high (up to $100 \mathrm{~nm}$ lateral resolution and up to $3 \mathrm{fps}$ operating speed) that makes this prospective technique tool for specific tasks in optical and microelectronic industries.

Acknowledgements. This work was financially supported by the Ministry of Education and Science of Russian Federation in the framework of the state task No 9.7453.2017/6.7.

The work is carried out on the equipment of the Center of collective use of MSTU "STANKIN".

\section{References}

[1] P.J. De Groot, Principles of interference microscopy for the measurement of surface topography, Adv. Opt. Photonics 7 (2015) 1-65

[2] F. Kaiser, P. Vergyris, et al., Quantum white-light interferometry for high-accuracy optical parameter determination, Quantum Inf. Meas. (QIM) QT6A.11 (2017)
[3] F. Liu, Y. Wu, F. Wu, Phase shifting interferometry from two normalized interferograms with random tilt phase-shift, Opt. Express 23 (2015) 19932-19946

[4] L. Huang, X. Lu, Y. Zhou, et al., Dual-wavelength interferometry based on the spatial carrier-frequency phase-shifting method, Appl. Opt. 55 (2016) 2363-2369

[5] T.A. Ramirez-delreal, M. Mora-Gonzalez, F.J. CasillasRodriguez, et al., Steps length error detector algorithm in phase-shifting interferometry using Radon transform as a profile measurement, Opt. Express 25 (2017) 7150-7160

[6] S. Mahajan, V. Trivedi, P. Vora, et al., Highly stable digital holographic microscope using Sagnac interferometer, Opt. Lett. 40 (2015) 3743-3746

[7] G. Nehmetallah, Multi-wavelength digital holographic microscopy using a telecentric reflection configuration, Digit. Hologr. 3D Imaging Meet. DM3A.7 (2015)

[8] P.S. Ignatiev, A.V. Loparev, K.V. Indukaev, P.A. Osipov, Investigating the optical properties of nanostructures by modulation interference microscopy, J. Opt. Technol. 78 (2011) 19-24

[9] A.V. Loparev, E.V. Romash, A.B. Zenzinov, et al., Laserbased modulation-interference microscopy of optical surfaces, J. Opt. Technol. 79 (2012) 366-370

[10] V.P. Tychinsky, Coherent phase microscopy in cell biology: visualization of metabolic states, Biochim. Biophys. Acta 1708 (2005) 362-366

[11] W. Kaplonek, C. Lukianowicz, Coherence correlation interferometry in surface topography measurements, in: I. Padron (Ed.), Recent Interferometry Applications in Topography and Astronomy, InTech, Chapters published, 2012

[12] A. Arias, M.G. Shlyagin, S.V. Miridonov, et al., Phasesensitive correlation optical time-domain reflectometer using quantum phase noise of laser light, Opt. Express 23 (2015) 30347-30356

[13] H. Lu, J. Chung, X. Ou, C. Yang, Quantitative phase imaging and complex field reconstruction by pupil modulation differential phase contrast, Opt. Express 24 (2016) $25345-25361$

[14] Z. Wang, D. Marks, S. Carney, M. Mir, G. Popescu, Tomographic reconstruction by quantitative phase imaging with broadband fields, Opt. Life Sci. NTuB2 (2011)

[15] N.I. Chkhalo, S.A. Churin, A.E. Pestov, et al., Roughness measurement and ion-beam polishing of super-smooth optical surfaces of fused quartz and optical ceramics, Opt. Express 22 (2014) 20094-20106

[16] Y.A. Melnik, T.V. Tarasova, G.O. Gvozdeva, S. Nowotny, High precision surface protection of Al-based alloy parts using laser micro cladding, Mechanics \& Industry 17 (2016) 710

[17] C. Li, Y. Yang, H. Chai, et al., Dark-field detection method of shallow scratches on the super-smooth optical surface based on the technology of adaptive smoothing and morphological differencing, Chin. Opt. Lett. 15 (2017) 081202

[18] L. Zhang, J. Wang, J. Zhang, Super-smooth surface fabrication technique and experimental research, Appl. Opt. 51 (2012) 6612-6617

[19] J. Lim, A. Wahab, G. Park, et al., Beyond Born-Rytov limit for super-resolution optical diffraction tomography, Opt. Express 25 (2017) 30445-30458 
[20] H. Yang, H. Cheng, Y. Feng, Improvement of high-power laser performance for super-smooth optical surfaces using electrorheological finishing technology, Appl. Opt. 56 (2017) 9822-9829

[21] N.I. Chkhalo, S.A. Churin, A.E. Pestov, et al., Roughness measurement and ion-beam polishing of super-smooth optical surfaces of fused quartz and optical ceramics, Opt. Express 22 (2014) 20094-20106

[22] R. Horstmeyer, J. Chung, O. Xiaoze, G. Zheng, Diffraction tomography with Fourier ptychography, Optica 3 (2016) $827-835$

[23] V.I. Teleshevskii, A.V. Shulepov, E.M. Rozdina, Smart computer microscopy for measurement of linear and angular dimensions of work pieces, Meas. Tech. 54 (2011) 853-858

[24] I. Smurov, M. Doubenskaia, S. Grigoriev, A. Nazarov, Optical monitoring in laser cladding of Ti6Al4V, J. Therm. Spray Technol. 21 (2012) 1357-1362
[25] I.S. Gershman, E.I. Gershman, P.Y. Peretyagin, Composite nanomaterials based on copper to replace silver in electrical contacts, Mechanics \& Industry 17 (2016) 708

[26] B. Bhaduri, C. Edwards, H. Pham, et al., Diffraction phase microscopy: principles and applications in materials and life sciences, Adv. Opt. Photon. 6 (2014) 57-119

[27] Q. Vo, F. Fang, X. Zhang, H. Gao, Surface recovery algorithm in white light interferometry based on combined white light phase shifting and fast Fourier transform algorithms, Appl. Opt. 56 (2017) 8174-8185

[28] S. Hurst, The characteristic function of the student- $t$ distribution, Financial mathematics, Centre for Mathematics and its applications, School of Mathematical Sciences, ANU, Canberra, Australia, 1995

[29] T. Doi, T. Kurosawa, T. Hatsuzawa, Estimation of numerical aperture effect on the basis of measured pupil function of Mirau-type Objective, Front. Opt. OFMC4 (2006)

Cite this article as: P.S. Ignatyev, A.A. Skrynnik, Y.A. Melnik, Nanoscale surface characterization using laser interference microscopy, Mechanics \& Industry 18, 713 (2017) 